\title{
EAGLE Spectroscopy of Resolved Stellar Populations Beyond the Local Group
}

\author{
Chris Evans ${ }^{1, a}$, Yanbin Yang ${ }^{2}$, Mathieu Puech $^{2}$, Matthew Lehnert ${ }^{2}$, Michael Barker ${ }^{3}$, Annette \\ Ferguson $^{3}$, Jean-Gabriel Cuby ${ }^{4}$, Simon Morris ${ }^{5}$, Gérard Rousset ${ }^{6}$, François Assémat ${ }^{6}$, and Hector \\ Flores $^{2}$ \\ 1 UK ATC, Royal Observatory Edinburgh, Blackford Hill, Edinburgh, EH9 3HJ, UK \\ 2 GEPI, Observatoire de Paris, 5 Place Jules Janssen, 92195 Meudon Cedex, France \\ 3 Institute for Astronomy, Royal Observatory Edinburgh, Blackford Hill, Edinburgh, EH9 3HJ, UK \\ 4 LAM, OAMP, 38 rue Frédéric Joliot Curie, 13388 Marseille Cedex 13, France \\ 5 Department of Physics, Durham University, South Road, Durham, DH1 3LE, UK \\ ${ }^{6}$ LESIA, Observatoire de Paris, 5 Place Jules Janssen, 92195 Meudon Cedex, France
}

\begin{abstract}
Valuable insights into galaxy evolution can be gleaned from studies of resolved stellar populations in the local Universe. Deep photometric surveys have provided tracers of the star-formation histories in galaxies from 0.8-16 Mpc, but without robust chemical abundances and stellar kinematics from spectroscopy, their substructures and assembly histories remain hidden from us. In this context, we introduce the EAGLE design study for a multi-integral-field-unit, near-infrared spectrograph for the European Extremely Large Telescope (E-ELT). EAGLE will exploit the unprecedented light-gathering power of the E-ELT to deliver AO-corrected spectroscopy across a large (38.5 $\left.\operatorname{arcmin}^{2}\right)$ field, truly revolutionising our view of stellar populations in the Local Volume.
\end{abstract}

\section{Introduction}

Discoveries of disrupted satellite galaxies have demonstrated that our evolutionary picture of the Milky Way is far from complete [1], let alone our understanding of galaxies elsewhere in the Universe. Deep imaging from ground-based telescopes and the Hubble Space Telescope (HST) has yielded colourmagnitude diagrams (CMDs) with unprecedented fidelity, providing new and exciting views of the outer regions of galaxies beyond the Milky Way for the first time, e.g. in M31 [2,3] and M33 [4]. From comparison with stellar evolutionary models, these data are used to explore the star-formation and chemical-enrichment histories of the targeted regions, providing a probe of the past evolution and, in particular, the merger/interaction histories of these external galaxies. There is increasing evidence for the accretion of numerous low-mass satellite galaxies in the assembly of the present-day Milky Way [5]. Do we see evidence for similar processes at work in other large galaxies? Moreover, what are the assembly histories in galaxies with very different morphological types, such as massive ellipticals, large metal-poor irregulars, and lower-mass, late-type spirals like M33?

Photometric methods are immensely powerful when applied to extragalactic stellar populations, but only via precise chemical abundances and stellar kinematics can we break the age-metallicity degeneracy, while also disentangling the populations associated with different structures, i.e. follow-up spectroscopy is required. Over the past decade the Calcium Triplet (CaT, spanning 0.85-0.87 $\mu \mathrm{m}$ ) has become an increasingly used diagnostic of stellar metallicities and radial velocities in nearby galaxies, providing new views of their star-formation histories and sub-structure, e.g. the Dwarf Abundances and Radial velocities Team (DART) large programme with VLT-FLAMES [6]. However, 8-10m class telescopes are already at their limits in pursuit of CaT spectra of the evolved populations in galaxies at distances greater than $\sim 300 \mathrm{kpc}$, e.g. Keck-DEIMOS observations in M31 struggled to yield useful signal-to-noise below the tip of the red giant branch at $I>21.5$ [7].

\footnotetext{
a e-mail: chris.evans@stfc.ac.uk
} the original work is properly cited. 


\section{Resolved Stellar Populations in the ELT Era}

With its vast primary aperture and excellent angular resolution, the E-ELT will be the facility to unlock spectroscopy of evolved stellar populations in the broad range of galaxies in the Local Volume, from the edge of the Local Group, out towards the Virgo Cluster. This will bring a wealth of new and exciting target galaxies within our grasp, spanning a broader range of galaxy morphologies, star-formation histories and metallicities than those available to us at present in the Local Group. These observations can then be used to confront theoretical models to provide a unique view of galaxy assembly and evolution. There are many compelling and ground-breaking targets for stellar spectroscopy with the E-ELT including, in order of distance:

- NGC 3109 and Sextans A with sub-SMC metallicities $\left(Z<0.2 Z_{\odot}\right)$, both at $1.3 \mathrm{Mpc}$.

- The spiral dominated Sculptor 'Group' at 2-4 Mpc.

- The M83/NGC5128 (Centaurus A) grouping at 4-5 Mpc.

- NGC 3379, the nearest normal elliptical at $10.8 \mathrm{Mpc}$.

- The Virgo Cluster of galaxies at 16-17 Mpc, the nearest massive cluster.

In contrast to proposed E-ELT observations of high-redshift galaxies, targets for CaT spectroscopy are readily available. For example, deep ground-based and HST imaging in galaxies in the Local Volume has begun to investigate their stellar populations [8-10], yet the stellar magnitudes are well beyond spectroscopy with existing facilities. Note that although we have focussed mostly on southernhemisphere targets here, there are equally compelling northern hemisphere targets, including the M81 group, and deeper studies in M31 and M33.

\section{EAGLE: A Multi-IFU, Near-IR Spectrograph for the E-ELT}

The EAGLE Phase A study is a French-UK partnership to provide an advanced conceptual design of an AO-corrected, near-infrared spectrograph with multiple integral-field units (IFUs). The specifications of the baseline design are summarised in Table 1, with the mechanical implementation shown in Figure 1. To minimise flexure on the instrument over the course of each night, EAGLE is intended to be located at the gravity-invariant focus of the E-ELT.

Table 1. EAGLE Baseline Design. The patrol field is the instrument field-of-view within which IFUs can be configured to observe individual targets.

\begin{tabular}{ll}
\hline Parameter & Specification \\
\hline Patrol Field & Eqv. $7^{\prime}$ diameter \\
IFU field-of-view & $1^{\prime \prime} 65 \times 1^{\prime \prime} 65$ \\
Multiplex (\# of IFUs) & 20 \\
Spatial resolution & $30 \% \mathrm{EE}$ in 75 mas $(H$ band $)$ \\
Spectral resolving power $(R)$ & $4,000 \& 10,000$ \\
Wavelength range & $0.8-2.5 \mu \mathrm{m}$ \\
\hline
\end{tabular}

Five top-level science topics have shaped the EAGLE design:

- Physics and evolution of high-redshift galaxies

- Detection and characterisation of 'first light' galaxies

- Galaxy assembly and evolution from stellar archaeology

- Star-formation, stellar clusters and the initial mass function

- Co-ordinated growth of black holes and galaxies

A summary of the high-redshift case and of E-ELT observations in the Galactic Centre region (combining elements of the last two) are given elsewhere in these proceedings $[11,12]$. Here we focus on simulated EAGLE performances for spectroscopy of resolved stellar populations, used to probe the assembly and evolution of their host galaxies. 


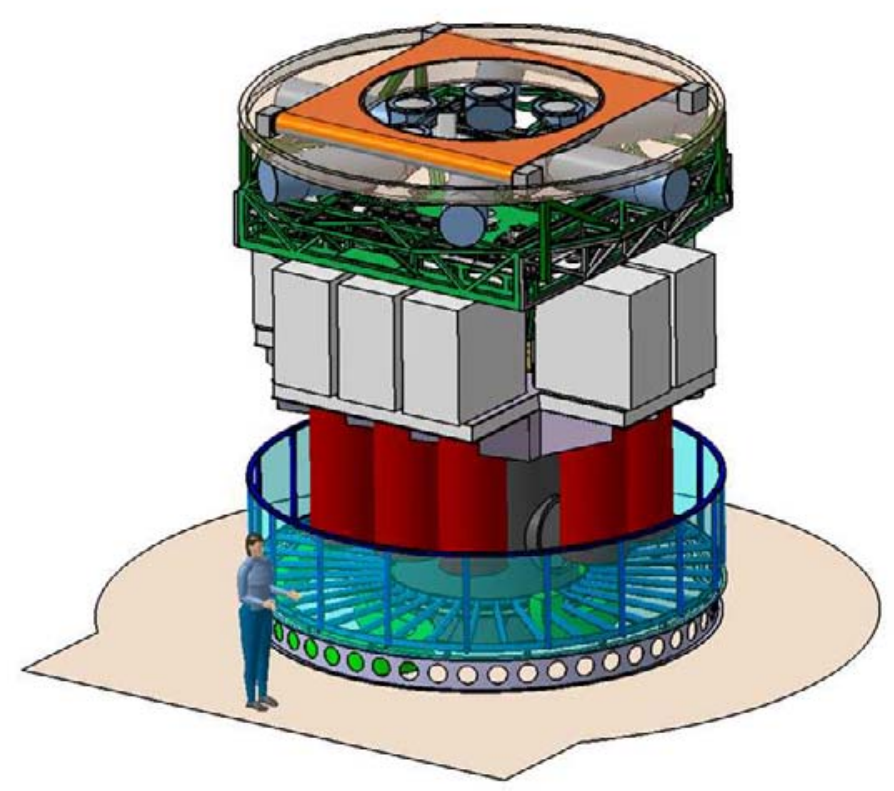

Fig. 1. Baseline mechanical design of EAGLE.

\section{EAGLE Adaptive Optics}

EAGLE will employ multi-object adaptive optics (MOAO) to provide significantly improved image quality for selected target fields within the focal plane [13, for example]. The EAGLE baseline design uses an array of six laser guide stars and five natural guide stars (NGS) to map the atmospheric turbulence. The deformable mirror in the telescope (M4) will be used to correct for the low-order wavefront error terms, with the high-order terms corrected by deformable mirrors in each science channel. An integral part of the EAGLE project is the CANARY on-sky demonstrator on the William Herschel Telescope in La Palma, discussed elsewhere in these proceedings [14].

The consortium has undertaken extensive simulations of MOAO performance, paying attention to the location and magnitude of potential NGS in real example fields [15]. Here we investigate the scientific peformance of EAGLE, making use of simulated point-spread functions (PSFs) from two NGS configurations ('\#10' and '\#1'), which are illustrative of relatively good and poor performance given the spatial distribution and magnitude of the available guide stars [15].

\section{EAGLE Performance: CaT Spectroscopy}

We have investigated EAGLE's performance in the CaT region, incoporating the new PSFs, using a modified version of the IFU simulation tool developed to characterise the MOAO requirements for observations of high-redshift galaxies [16]. The principal assumptions in the CaT simulations were:

- Telescope: 42-m primary, with central obscuration of $9 \%$

- Exposure time: $10 \mathrm{hrs}(20 \mathrm{x} 1800 \mathrm{~s})$

- $\lambda$-range: $8400-8750 \AA$, and $R=10,000$

- Spatial sampling: 37.5 mas spatial pixels

- Total throughput: telescope (0.8), atmosphere (0.95), instrument \& detector (0.25@ 0.85 $\mu \mathrm{m}): 0.19$

- Detector read-out noise: $5 \mathrm{e}^{-} /$pixel

- Dark current: $0.01 \mathrm{e}^{-} / \mathrm{pixel} / \mathrm{s}$ 
An example CaT spectrum $\left(\mathrm{T}_{\text {eff }}=4,000 \mathrm{~K}, \log g=2.0\right)$ is adopted as a template from the synthetic spectra calculated using Kurucz model atmospheres for the GAIA mission [17]. The synthetic spectra were calculated for $R=20,000$, i.e. sufficiently over-sampled so as to be degraded to either of the two spectral resolving powers provided by EAGLE. To this template we append an additional continuum region to provide a line-free region with which to investigate to the signal-to-noise $(\mathrm{S} / \mathrm{N})$ of the spectra in the resulting datacubes.

Assuming a Paranal-like site, we have investigated two sets of seeing conditions in the simulations: 0.65 at $\lambda=0.5 \mu \mathrm{m}$ at zenith (the mean VLT seeing at Paranal, [18]) and $0{ }^{\prime} 90$ at a zenith distance (ZD) of $35^{\circ}$, providing a good investigation of the performance from execution of a 'Large Programme'like survey. The S/N results for the two NGS configurations are given in Table 2 . These results are for spectra extracted from the central spatial pixel of a point source at the centre of the cube (optimal PSFfitting extractions should be able to improve on these). The key result is that $S / N \geq 10$ is recovered from a stacked $10 \mathrm{hr}$ exposure at $I=24.5$, in mean seeing, in both NGS configurations. This corresponds to spectroscopy of stars at the tip of the red giant branch (RGB), with $\mathrm{M}_{I}=-4$, out to $\sim 5 \mathrm{Mpc}$ in just $10 \mathrm{hrs}$. This is four magnitudes deeper than FLAMES-GIRAFFE using the LR08 grating $(R=6,500)$ with the same exposure time.

Table 2. EAGLE CaT results: Continuum $\mathrm{S} / \mathrm{N}$ obtained for $R=10,000, t_{\mathrm{exp}}=10 \mathrm{hrs}$.

\begin{tabular}{ccccc}
\hline & \multicolumn{2}{c}{ Seeing $=0$. '9 @ ZD $=35^{\circ}$} & \multicolumn{2}{c}{ Mean VLT Seeing $\left(0.65\right.$ @ $\left.\mathrm{ZD}=0^{\circ}\right)$} \\
$I_{\text {VEGA }}$ & NGS ‘good' & NGS ‘poor' & NGS 'good' & NGS ‘poor' \\
\hline 22.5 & 40 & 27 & 56 & 48 \\
23.5 & 16 & 11 & 28 & 24 \\
24.5 & 8 & 4 & 13 & 10 \\
\hline
\end{tabular}

Some high-order contributions to the wavefront error, as well as contributions from tip-tilt and defocus, are not included in the simulated PSFs [15]. Scaling the PSFs for this in science simulations is non-trivial as the flux will be conserved (re-distributed) rather than lost. In additional simulations the PSFs were artificially scaled by a further throughput factor $(0.65)$ to give a pessimistic example for a given turbulent profile, seeing etc., still yielding $\mathrm{S} / \mathrm{N} \sim 10$ for $I=24.5$ in the good NGS configuation, in mean seeing, in $10 \mathrm{hrs}$

Although we have focussed on the $R=10,000$ case here, similar calculations at $R=4,000$ for $I=24.5$ and 26.0 (with the latter corresponding to approximately the tip of the RGB in NGC 3379) yield $\mathrm{S} / \mathrm{N} \geq 10$ in 5 and $80 \mathrm{hrs}$, respectively.

\section{EAGLE Observations in the Sculptor Group}

An example Large Programme for EAGLE is to undertake spectroscopy of evolved stars in the galaxies in the Sculptor Group, comprising five spirals (NGC 55, NGC 247, NGC 253, NGC 300 and NGC 7793) and numerous dwarf irregulars. Distance estimates over the past decade have revealed that this 'group' is actually two distinct components [19], at approximately $1.9 \mathrm{Mpc}$ (NGC $55 \& 300$ ) and 3.6-3.9 Mpc (NGC 247, 253 \& 7793). These five galaxies represent the most immediate opportunity to study the star-formation history and mass assembly of spirals beyond the limited sample available at present, i.e. the Milky Way, M31 and M33. Their masses are in the range $1.5-8 \times 10^{10} \mathrm{M}_{\odot}$, putting them on a par with M33 - it is exactly these late-type, low-mass, small bulge (or even bulge-less) spirals which are the systems that theoretical N-body/semi-analytic simulations struggle hardest to reproduce [20].

We propose spectroscopy of stars in the upper RGB, spanning $\mathrm{M}_{I}=-4$ (at the tip of the RGB) to approximately $\mathrm{M}_{I}=-2$, i.e. $22.5<I<24.5$ in NGC 55 and NGC 300, and down to $I \sim 24.5$ (i.e. $\left.\mathrm{M}_{I} \sim-3\right)$ in the others. Targets would be observed along the major and minor axes of each galaxy, sampling the stellar populations across different spiral structures and the halos. For instance, NGC 55 

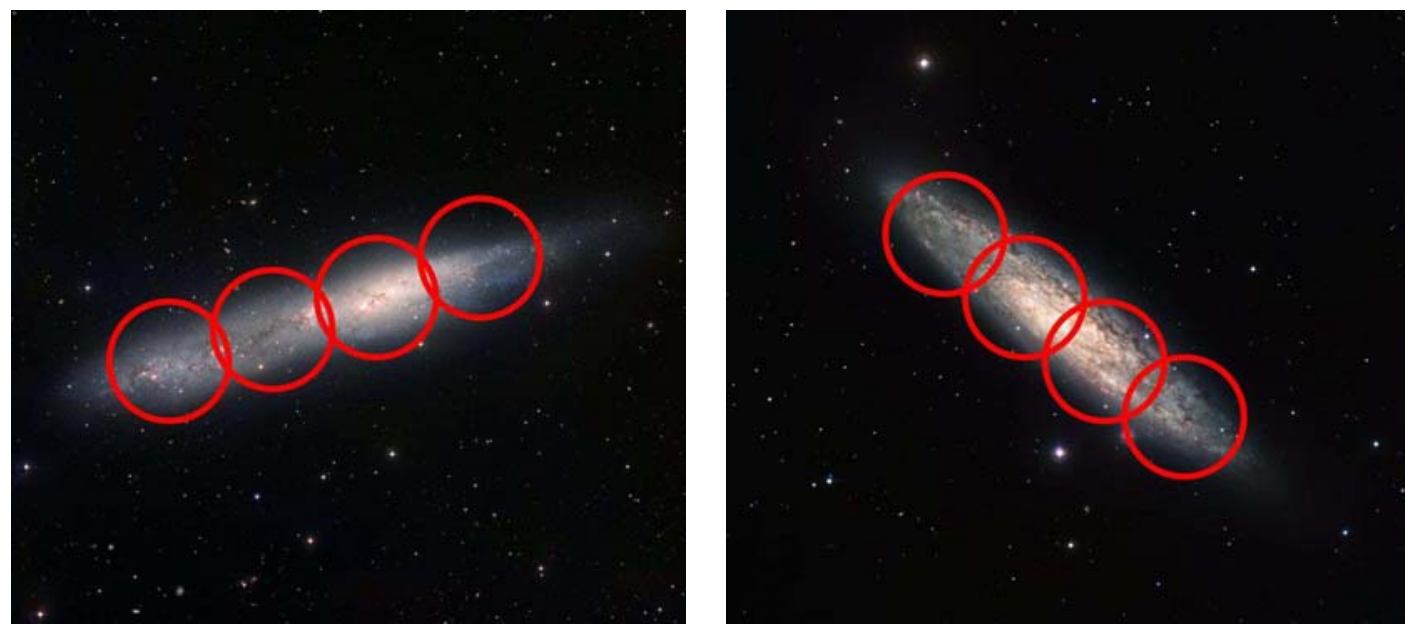

Fig. 2. Example major-axis EAGLE pointings in NGC 55 at $1.9 \mathrm{Mpc}($ left $)$ and NGC 253 at $3.6 \mathrm{Mpc}$ (right). The central (unvignetted) 5 arcmin field of EAGLE is illustrated by the red circles. [Original image credits: ESO].

and NGC 253 both have semi-major axes greater than ten arcminutes, i.e. multiple EAGLE pointings are required to survey stars across the full extent of such a galaxy, as shown in Figure 2. In all but the most rarefied regions, multiple stars will be observed with each IFU (thus boosting the effective multiplex), with the IFUs also enabling better sky subtraction compared to fibre/slit methods. Adopting $10 \mathrm{hrs} /$ pointing for NGC 55/NGC 300 and $15 \mathrm{hrs} /$ pointing for the other three galaxies (cf. the sensitivity results in Table 2), well in excess of 1,000 stars can be observed in a total of $\sim 240 \mathrm{hrs}$.

The left-hand panel of Figure 3 shows a typical $1^{\prime \prime} \times 1^{\prime \prime}$ IFU pointing in the central region of NGC 55 (in which the simulations adopted a smaller IFU than in the baseline design simply to reduce the computation time). The magnitudes and relative positions of the stars are from HST Advanced Camera for Surveys (ACS) observations in the core region of NGC 55, taken as part of the GHOSTS survey [9]. This example illustrates perfectly the gain in effective multiplex from the IFUs (i.e. nine stars in this $1^{\prime \prime} \times 1^{\prime \prime}$ region), with minimal impact from crowding. A simulated CaT spectrum $(I=23.5$, $\mathrm{S} / \mathrm{N} \sim 25)$ is shown in the right-hand panel of Figure 3.

Recent simulations predict that stellar radial mixing (also called radial 'churning', or orbit switching) due to perturbations from transient spiral density waves plays a large role in shaping the age and metallicity gradients [21] - this dynamical process could have huge implications for stellar archaeology, as it may modify, or even erase, the original gradients. Ages and metallicities will be derived for each star to investigate the gradients of these properties across each galaxy, while also inspecting the results for evidence of sub-structure. In outer halo fields we will also test the prediction that halo stellar metallicity is thought to scale with halo stellar mass $[22,23]$.

\section{Summary}

The EAGLE design provides a unique combination of abilities to harness the power of the E-ELT for spectroscopy of resolved stellar populations. The image quality from MOAO will be significantly better than that obtained from seeing-limited or ground-layer AO modes, enabling us to explore spatiallyresolved, extragalactic stars across wide fields of over five arcminutes.

EAGLE spectroscopy in the Sculptor Group is just one illustration of a potential Large Programme. Even within the Sculptor galaxies one might prioritize, e.g., NGC 253 (due to its starburst activity) or NGC 300 (due to its relative proximity) first, building-up a comprehensive survey in one galaxy or following-up on interesting sub-structures before turning to the others. Moreover, deeper observations of the most luminous evolved stars in selected fields in galaxies at $10 \mathrm{Mpc}$ and beyond will also be hugely attractive. 

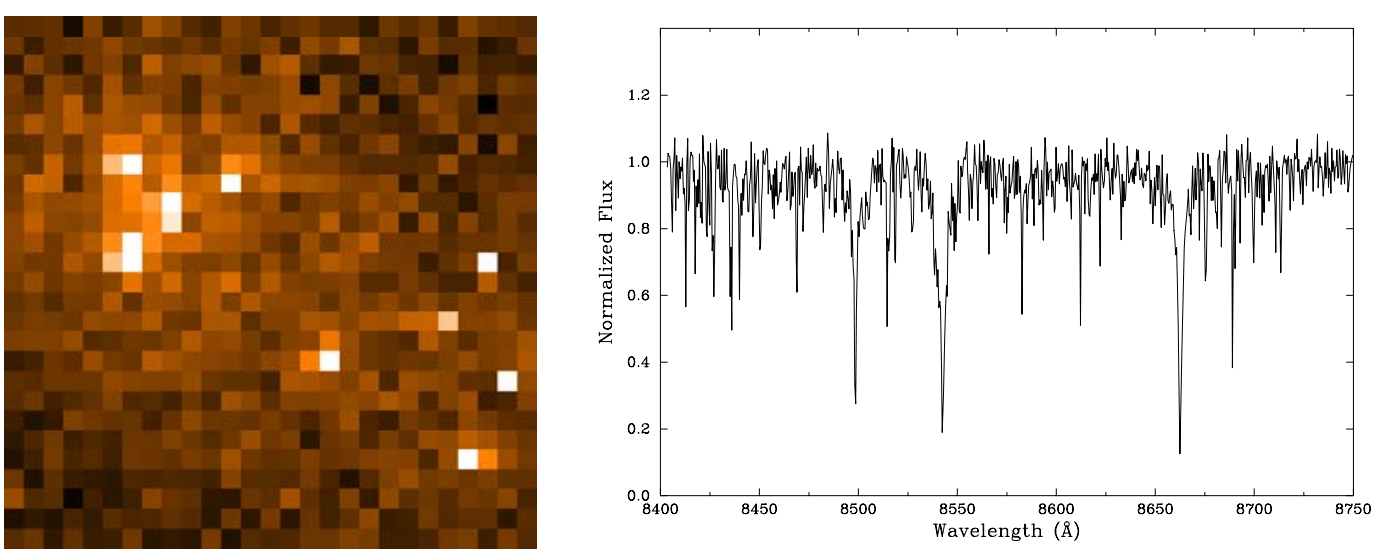

Fig. 3. Left: Front slice of simulated IFU datacube for EAGLE observations in the central region of NGC 55; Right: Simulated CaT spectrum for a star with $I=23.5$, yielding $\mathrm{S} / \mathrm{N} \sim 25$ in the continuum.

\section{References}

1. Majewski, S. R., Skrutskie, M. F. , Weinberg, M. D. \& Ostheimer, J. C., ApJ 599, 2003, 1082

2. Ferguson, A. M. N. et al., ApJL 622, 2005, 109

3. Richardson, J. C. et al., AJ 135, 2008, 1998

4. Barker, M. K. et al., AJ 133, 2007, 1125

5. Belokurov, V. et al., ApJL 642, 2006, 137

6. Tolstoy, E. et al., ApJL 617, 2004, 119

7. Chapman, S. et al., ApJ 653, 2006, 255

8. Rejkuba, M. et al., ApJ 631, 2005, 262

9. de Jong, R. S. et al., IAUS 241, 2007, 503

10. Dalcanton, J. J. et al., ApJS 183, 2009, 67

11. Puech, M. et al., these proceedings

12. Paumard, T. et al., these proceedings

13. Assémat F., Gendron E., Hammer F., MNRAS 376, 2007, 287

14. Morris, T. et al., these proceedings

15. Rousset, G. et al., these proceedings

16. Puech, M. et al., MNRAS 390, 2008, 1089

17. Munari, U. \& Castelli, F., A\&AS 141, 2000, 141

18. Sarazin, M., Melnick, J., Navarrete, J. \& Lombardi, G., Msngr 132, 2008, 11

19. Karachentsev, I. D. et al., A\&A 404, 2003, 93

20. D’Onghia, E. \& Burkert, A., ApJ 612, 2004, L13

21. Roskar, R. et al., ApJ 684, 2008, L79

22. Mouhcine, M. et al., ApJ 633, 2005, 821

23. Font, A. S. et al., ApJ 646, 2006, 886 